\title{
CONSIDERAR LO RELIGIOSO: CONTRIBUCIONES PARA SU ESTUDIO EN LAS CULTURAS CONTEMPORÁNEAS
}

\section{TO CONSIDER RELIGION: CONTRIBUTIONS FOR ITS STUDY IN CONTEMPORARY CULTURES}

\section{Patricio Ivan Pantaleo*}

\section{RESUMEN}

El artículo aborda las principales cuestiones teórico-metodológicas para el estudio de la religión con el objetivo de aportar a la complejización analítica y a la valoración de la religión como un ámbito principal de acción y re-significación de los sentidos que repercuten en la dinámica político-cultural de las sociedades actuales. Para ello, se realiza una doble diferenciación; por un lado, los planteamientos teórico-filosóficos que implica pensar lo religioso como fenómeno o experiencia; por otro, las principales corrientes de estudio de la religión en el siglo xx.

PALABRAS CLAVE: RELIGIÓN * ESTUDIOS CULTURALES *FILOSOFÍA* EXPERIENCIA RELIGIOSA *EPISTEMOLOGÍA

\section{ABSTRACT}

This article deal with the main theoretical and methodological issues in the study of religion, with the purpose of contributing in the analytical complexity as well as in the valoration of the religion as a major scope of action, taking into account the re-signification of the meanings involved in the cultural and political dynamics of present societies.To achieve this, it takes a double differentiation. On the one hand, theoretical and philosophical positions that implicate thinking about religion as a phenomenon ora experience. On the other, mainstreams of religion studies in the twentieth century.

KEYWORDS: RELIGION * CULTURAL STUDIES $*$ PHILOSOPHY * RELIGIOUS EXPERIENCE * EPISTEMOLOGY

Universidad Nacional de Río Cuarto, Argentina.

patrickevin@yahoo.es 


\section{INTRODUCCIÓN}

El presente artículo tiene como objetivo primordial abordar las principales cuestiones teórico-filosóficas suscitadas en torno al estudio de la religión y su implicancia para la sociedad contemporánea, en el marco de los quiebres epistemológicos que se dan en el tránsito de los paradigmas del siglo xix, así como, su progresivo abandono y crítica en la primera mitad del siglo xx.

En este contexto, se plantean relaciones ambivalentes. En primer lugar, se varía desde una caracterización meramente fenomenológica de lo religioso específicamente en el siglo xix, hasta una complejización que considera lo experimental y la vivencia propia de los protagonistas por sobre la descripción y consideración meramente racional y académica de la religión. Por otra parte, este transcurso gnoseológico desde la observación a la experiencia, muestra un correlato en los principales abordajes teóricos que se realizan en las academias del momento. Entre los principales representantes de estas, se puede ver a quienes van desde un ateísmo analítico al excentricismo antropológico, junto también a una postura frente a la diversidad religiosa por parte de las instituciones eclesiásticas que se encuentran entre un evangelismo pastoral hasta en un dogmatismo inquisidor.

Intentando superar estos extremos, las discusiones histórico-culturales empiezan a revalorizar a la religión como un proceso complejo, constitutivo y elocuente de los procesos de significación humana, brindando así nuevas posibilidades de estudios que aporten al diálogo y al conocimiento cultural a través de la valoración de experiencias religiosas diversas.

Para cumplir con el objetivo propuesto, el desarrollo utiliza como herramienta metodológica la siguiente hipótesis: el estudio de lo religioso se caracteriza, gnoseológicamente y en el auge de la Modernidad, por una preponderancia de estudios meramente fenomenológicos; esto muestra su correlato en una actitud del ámbito intelectual-académico que repliega su valoración de lo religioso a lo puramente descriptivo $y$ en muchos casos, a lo irracional y pre-científico (en su connotación peyorativa, es decir, como un discurso que no puede dar cuenta de la realidad en ninguna de sus aristas). Frente a esto, entrado el siglo xx, con el desarrollo de los campos históricos y sociológicos de la cultura, lo religioso empieza a ser concebido como una experiencia que da cuenta de elementos esenciales de la cultura humana en la que está inserto y que su estudio puede aportar, en los espacios académicos, a un entendimiento más complejo de la dinámica social.

Se dividirá la estructura del escrito en dos acápites que dan cuenta de las principales cuestiones teóricas y sus abordajes. El primero de ellos indaga en la naturaleza de lo religioso entendido como "fenómeno", en sus diferentes acepciones, asimismo, se diferencia y problematiza con las consideraciones que lo entienden como experiencia. Una vez brindadas las principales consideraciones categoriales, el segundo capítulo manifiesta las consideraciones a las que se somete el estudio de la religión, en tanto los principales paradigmas epistemológicos que la abordan durante la crisis de la Modernidad. Se profundiza, en primer lugar, cómo se abordó el estudio de lo religioso entre ateos analíticos y dogmáticos religiosos en el marco de descripciones fenomenológicas etnocéntricas y decimonónicas que perduran, en algunos ámbitos, hasta la actualidad. Por último, se aborda como se llega a una valoración e inclusión de lo religioso en los estudios histórico-culturales de la primera mitad del siglo xx, dando fuerte hincapié a la experiencia y sus múltiples manifestaciones sociales, hasta la relevancia que adquiere en los estudios interdisciplinares actuales.

\section{FENÓMENO Y EXPERIENCIA. CARACTERIZACIÓN COMPLEJA DE LO RELIGIOSO}

Frente a la pregunta ¿cuál es la naturaleza de lo religioso?, surgen a lo largo de la historia diferentes respuestas, dependientes cada una de la postura que se toma frente al fenómeno religioso en sí y a la religión. Por su parte, el concepto de "fenómeno" aplicado a la experiencia religiosa puede presentar algunos inconvenientes. La experiencia religiosa es variada y en algunos casos, busca dar 
comprensión al hombre de lo trascendental, de lo absoluto, más allá de lo a priori percibido. Por esa razón, esta experiencia calificada meramente como "fenómeno" no expresa la complejidad a la cual puede dar acceso. En otras palabras, identificar como "fenómeno" a una experiencia que busca dar cuenta de lo trascendente, es contradictorio.

Es posible identificar dos acepciones principales de "fenómeno". Por un lado, entendido como manifestación que se hace consciente en el sujeto, meramente como un objeto de su percepción, donde la cosa en sí permanece siempre oculta e incognoscible. En este caso, esta concepción siempre alude a un estudio y un sentido del "fenómeno religioso" como una manifestación fenoménica, por lo que se concibe un acceso limitado $y$ meramente descriptivo del sujeto a la esencia misma de la cosa en sí. Por otro lado, si se adopta una postura fenomenológica post-husserliana, es posible identificar la intencionalidad que subyace a la descripción del fenómeno, como una posibilidad de acceso a las esencias puras, trascedentes a la misma conciencia del sujeto, pero siempre a través de lo percibido del fenómeno. La indagación en el fenómeno a priori percibido, posibilitaría aquí, un conocimiento de la esencia, oculta tras los sentidos. El fenómeno religioso facilitaría un acceso a su esencia, pero siempre mediante la profundización de su apariencia.

Ambas acepciones mencionadas parten, desde este análisis, de un paradigma gnoseológico desarrollado en Occidente, donde las percepciones sensoriales brindan un acceso o bien, al fenómeno o a su esencia. Sin embargo, a diferencia de esto, muchas religiones orientales se cimentan sobre pre-supuesto gnoseológicos distintos, que hacen difícil la utilización del término "fenómeno" para el análisis de estas experiencias religiosas específicas. Esto es así, porque su objetivo no es permanecer en el "fenómeno" o en las percepciones, sino, en todo caso, diluirlo y acceder a lo esencial. Parten de una negación misma de que lo percibido sea "real" y que pueda brindar un acceso a la esencia. A modo de ejemplo, se puede decir que gran parte del desarrollo doctrinal del Buddhismo parte de que las percepciones humanas y el fenómeno en sí, es ilusión, es māyā, por lo que no da una categoría de "real" a todo lo percibido a priori por los sentidos humanos y el acceso a la "realidad última" muchas veces - sino todasestá posibilitado por una contradicción fundamental del pensamiento lógico-racional $y$ de las percepciones básicas de la experiencia. El kōan de la tradición zen, es un ejemplo de ello. Todo esto denota la complejidad de la utilización del término "fenómeno" para la experiencia religiosa, ya que esta encierra diferentes $y$ a veces contradictorias formas de percibir y acceder a "lo real", que el término "fenómeno", como se dijo, no expresa.

Ahora bien, denotada esta complejización de la característica fenoménica de la experiencia religiosa, cabe indagar en la comprensión de la experiencia en sí. Por su parte, Pikaza asocia el fenómeno religioso a la experiencia humana. A tal fin, sostiene:

Con cierta frecuencia se asocia el fenómeno religioso a las experiencias paranormales o psicológicamente extrañas, como si lo divino debiera vincularse a lo extra-racional o raro. Otras veces se identifica la religión con los fenómenos esotéricos, con la mística en lo que puede tener de vivencia humana el misterio. En contra de eso, quiero situar la religión en el centro de la experiencia humana $(1999,21)$.

En esta afirmación, el autor asienta que toda experiencia humana es preferiblemente racional y que la religión es susceptible de situarse en esta experiencia racional (en contraposición a los "extra-racional o raro"). Si bien se concuerda con la afirmación de que la experiencia religiosa es humana en tanto racional, se agrega que no solo la experiencia humana es racional $y$ por ende, tampoco la experiencia religiosa es solamente racional. Se parte aquí de que el ámbito de la experiencia humana $y$ las percepciones, las vivencias y el acceso a la esencia de ellas, como anteriormente se mencionó, puede ser posibilitada —así lo es en muchas cosmovisiones no occidentales- por medios que no son específicamente racionales, sino que implican más bien una superación o trascendencia de ellos. Puede identificarse 
este proceso, no como irracional, sino más bien, como "meta-racional". La experiencia es definida por Pikaza como "...la manera específicamente humana de conocer, en un proceso de encuentro activo y receptivo con el mundo, con la propia vida y con la existencia de los otros" (1999, 22). Así, dentro de este proceso humano, la comprensión no solo se realiza de manera racional, sino que se da también intuitiva, motriz y trascendentalmente en una complejidad gnoseológica.

A esto apunta, en parte, la reelaboración heideggeriana del concepto de comprensión, que ayuda a una superación del paradigma cognitivo clásico. Supone entender a esta, no como una forma posible de conocimiento, sino como un fenómeno fundamental de la experiencia humana. En palabras de Apel, se pasa de la tesis de que "la comprensión solo tiene una función auxiliar heurístico-psicológica en el contexto de descubrimiento", a la comprensión "como modelo del humano "ser-en-el-mundo»" $(1985,24)$. Sin embargo, el mundo y nuestra comprensión de ello, se inscribe siempre dentro del fenómeno, “...irreductible a mis esfuerzos de búsqueda y trabajo; su ser-ahí precede a mis razones" (Pikaza 1999, 61-62). Así, la cuestión está en identificar a la experiencia religiosa con su intencionalidad, para poder delimitar como nos relacionamos con lo percibido y la naturaleza en sí de lo que la experiencia puede brindar.

Subyacen tres tipos de intencionalidad. En primera instancia, la intención de que mediante la experiencia religiosa, se acceda a una comprensión íntegra del fenómeno. En segundo lugar, que la experiencia religiosa y mediante la profundización de lo fenoménico, brinde un acceso a lo esencial o trascendental. Por último, que mediante la negación de la manifestación fenoménica y la percepción cotidiana, se brinde un acceso vivencial a lo esencial. Las dos primeras son propias de la experiencia religiosa Occidental, caracterizada por un fuerte conocimiento racional. La indagación en el fenómeno o buscar lo esencial, es posibilitado e indagado mayoritariamente mediante la razón. Ahora, la última intencionalidad de la experiencia religiosa adhiere medios al re-conocimiento de lo esencial tras el fenómeno. Es característico de las tradiciones principalmente orientales o también se puede encontrar en la tradición apofática de la teología cristiana; es decir, el acceso a lo trascendental mediante la negación.

Con base en el análisis, se puede deducir las siguientes premisas para continuar con el desarrollo. Las categorías analíticas del pensamiento occidental, presentan insuficiencias al momento de dar cuenta de la complejidad de la experiencia religiosa. La experiencia es variada, nunca uniforme $y$ depende de la intencionalidad que subyace en cada una de ellas. Por su parte, la intencionalidad varía dentro de las experiencias religiosas según sea su procedencia. Por todo esto, es que se denota la complejidad de dicha experiencia y la diversidad que posibilita los medios de acceso.

Ahora bien, Pikaza (1999) expone tres modelos de religión de acuerdo a tres caminos de experiencia de lo religioso. En estos y haciendo expresa la complejidad más allá de las categorías, el lenguaje simbólico y el lógico se alternan como medio de expresión. De los tres modelos, uno es el que pertenece a una forma de experiencia radicalmente diferente, donde el cosmos entero es expresión de lo divino, mientras que en las otras dos, el hombre supera al cosmos, $y$ en su propia subjetividad indaga y realiza o no, la manifestación divina. Ambas están divididas cronológicamente por lo que Jaspers (1968) llama el tiempo-eje. Este es un período, aproximadamente entre el $800 y$ el 200 a.C., donde la experiencia religiosa y la comprensión del lugar que el hombre tiene en ella $y$ en el cosmos, cambian radicalmente. En la religiosidad anterior a este cambio, llamada por Pikaza (1999) como "religiones cósmicas", el cosmos es expresión entera del misterio divino y el hombre, sujeto a dicho orden, interacciona de manera exterior y pasiva en la experiencia religiosa. Este autor señala como común "el hecho de que el cosmos aparece como todo numinoso y los humanos se realizan penetrando en su misterio" (1999, 162). Luego del tiempo-eje, se consolida una nueva forma de relación con lo divino y por ende, una nueva forma de experiencia; sin suponer específicamente que la anterior desaparezca por completo, sino que más bien, disminuye ante lo 
nuevo. El tiempo-eje presenta cierta antropologización en esta situación. El hombre puede encontrar ahora lo divino en su propia vida $y$ en su interioridad, dejando de someterse a la naturaleza y al orden cósmico específicamente. La novedad del tiempo-eje es la espiritualización:

...el ser humano puede oponerse interiormente al conjunto del mundo, porque descubre en su interior una base 0 fuerza que le capacita para alzarse sobre sí mismo y el mundo. Antes, el ser humano vivía bajo una especie de esclavitud cósmica: atado a la naturaleza. Después se ha liberado, descubriendo la posibilidad de ser él mismo y de encontrar lo divino en el proceso de su propia vida humana (Pikaza 1999, 164).

De este proceso surgen dos caminos de realización y de experiencia religiosa. Por un lado, en el mundo hindú principalmente, donde surge lo que Pikaza llama "religiones de la interioridad", las cuales centran la vivencia religiosa en la búsqueda interior de lo divino, mediante una negación de la exterioridad y de lo percibido. Según Pikaza, son de carácter místico "y buscan a Dios (lo sagrado) en el mismo proceso de purificación, profundidad y equilibrio interior de los humanos" $(1999,162)$. Por otro lado, el tiempo-eje da lugar a una religiosidad ocupada del tiempo y de la historia como posibilidad de salvación y manifestación de lo divino. Estas son las religiones de la historia, donde la experiencia religiosa es una vivencia exterior pero no cósmica, sino del hombre o su comunidad en relación con lo divino. Es desarrollada principalmente en Israel, donde Dios es salvación futura de las calumnias existenciales:

...acentúan el aspecto profético y descubren la palabra y presencia de Dios en el mismo camino de creatividad social del ser humano... Es un Dios fuerte, que habla $y$ dirige de forma personal la historia humana. Pero, al mismo tiempo, es un Dios débil, pues no puede ya identificarse con los fenómenos del cosmos ni tampoco con la transparencia interior de unos expertos religiosos (Pikaza 1999, 163).
En estos tres tipos diferentes de experiencias religiosas, el lenguaje simbólico actúa como principal medio de expresión de la complejidad que los representa. Complejidad en tanto que no es posible en ni una de ellas, acceder a lo que se considera como divino, de una manera meramente racional. Siempre implica una trascendencia que, si bien no es accesible en su totalidad mediante la razón, no por ello deja de manifestarse al humano. Lo que la razón no logra captar y sistematizar es, tal vez, la mayor parte de la manifestación divina; la cual se vale para expresarse, del simbolismo, dentro de todas sus variantes.

Eliade sostiene respecto del símbolo, que este es en sí mismo una hierofanía y disfruta de un lugar primordial en la experiencia religiosa:

... revela una realidad sagrada o cosmológica que ninguna otra 'manifestación' puede revelar. El simbolismo continúa la dialéctica de la hierofanía transformando los objetos en otra cosa distinta de lo que parecen ser en la experiencia profana (citado en Allen 1985, 134).

El símbolo expresa y da a conocer al mundo humano lo trascendente, mediante una comprensión meta-racional. Es la naturaleza compleja de la experiencia religiosa que obliga a lo sagrado a expresarse mediante símbolos: “... apuntan más allá de sí mismos, y adquieren significaciones que no son ni directas, ni literales, ni corrientes" (Allen 1985, 136).

Así, el simbolismo religioso está presente de diferentes maneras, en los tres tipos de experiencias religiosas anteriormente mencionadas, expresando en parte lo común de estas, que es su trascendentalidad y la complejidad de manifestar lo sagrado en un lenguaje humano; así como, lo diferente, que consiste en las distintas manifestaciones arquetípicas condicionadas por el contexto histórico-cultural, que lleva a la expresión simbólica a adquirir diferentes signos de expresión. La experiencia religiosa así, "desborda el plano de la mera conciencia" (Allen 1985, 216) y son los símbolos los principales comunicadores de dicha característica: “... pueden revelarnos una estructura del mundo que no es evidente en el plano de 
la experiencia inmediata" (Allen 1985, 156). La diferenciación no reside, como se mencionó, entre lo simbolizado, sino en la expresión figurativa del símbolo, que está condicionado y modificado por el contexto histórico-cultural en el que se elabora.

Una vez brindadas las principales consideraciones respecto del ámbito de análisis que al escrito compete, se aborda a continuación las principales corrientes que han abordado el estudio de la religión en el quiebre de la modernidad epistémica.

\section{LA RELIGIÓN Y SU ESTUDIO. RESEÑA SOBRE LOS PRINCIPALES ABORDAJES}

El proyecto de la Modernidad decimonónica inscribe en el concepto de "cultura" una acepción que es elitista y eurocéntrica, basada en una concepción cartesiana del sujeto, valorativa de la razón occidental como canon de inteligencia y de su expresión técnica como civilización. El sujeto cartesiano que se ocupa de discernir entre lo verdadero y lo falso, lo necesario y lo inútil, lo bueno y lo malo, se expresa en los primeros años del siglo xx, en un discurso hegemónico que necesita para su legitimación, la trivialización de otros. El discurso científico positivista expresa claramente la definición de sujeto que Hopenhayn aborda:

Es aquel que se atribuye cualidades intrínsecas que permiten discernir entre el conocimiento verdadero $y$ el falso, $y$ entre lo real y lo aparente; que se percibe como indisoluble en su identidad y consistente en sus convicciones; que cree conocer la racionalidad de la historia ( $y$ de su historia personal) y deducir de allí la capacidad para guiar esas mismas historias; $y$ que se declara sujeto 'trascendental' por cuanto se presume dotado de una moral de validez universal, o de la facultad para remontar el conocimiento de la realidad hasta sus últimas razones (1997, 11).

Partiendo de esta forma determinada de interpretar lo que se conoce (a priori cognitivo), la religión es abordada a fines del siglo
xIx $y$ principios del xx como una mera manifestación de la irracionalidad humana, como un intento de explicación de fenómenos naturales o sociales sin la debida profundización en la dilucidación de las razones suficientes que le dan origen. Frente a esto, el hombre carente de desarrollo racional desliga en una fuerza metafísica trascendental, las razones ocultas que no es capaz de develar por medio del propio intelecto, permaneciendo sin salir de la "minoría de edad", recurriendo a modelos autoritarios que sustituyan las representaciones de su infantil intelecto. La Modernidad subyuga la búsqueda de sentido, que se expresa en concepciones trascendentales, a una mera manifestación de la incapacidad racional del hombre. Pikaza sostiene al respecto:

Antiguamente, los trascendentales (verum, bonum, pulchrum; verdad, bondad, belleza) se entendían como función del ser, notas primigenias de toda realidad, $y$ debían apoyarse en lo divino. Desde la Ilustración y Kant, ellas se muestran como un simple momento de la relación del humano con el mundo. No valen por sí mismas, son función de la cultura. Por eso, la religión deja de entenderse como resultado de una revelación trascendente y aparece como simple creación racional y/o irracional del ser humano $(1999,80)$.

Esta acepción del fenómeno religioso es amplia y presenta matices respecto de los pensadores que se estudien, sin embargo desde el empirismo de Hume, quien rechaza la existencia de lo trascendente pero reconoce el sentimiento como función orientadora de la vida, pasando por el materialismo de Feuerbach y de Marx hasta el positivismo de Comte, que le resta a la religión la validez de su explicación de lo real pero reconoce el poder político de la misma para dar una "... organización general a la humanidad" (Cipriani 2011, 47), se desarrollan corrientes en las que prima una negación de la validez (no solo universal, sino también particular) del discurso religioso. Se afianzan así, perspectivas analíticas que tienden cada vez más a negar lugar a la religión en los estudios 
sobre la sociedad, dejando solo una puerta abierta al desarrollo antropológico que tenía más la intención de coleccionar creencias que consideraba "exóticas" para exponerlo en los museos europeos, que a dilucidar $y$ valorar la diversidad de sistemas de sentidos y creencias elaborados en distintas partes histórico-geográficas.

Por otra parte, en la Modernidad también se expresa, de herencia medieval $y$ en directo conflicto con las acepciones analíticas, abordajes del fenómeno religioso, específicamente dogmáticos que no solo concebían a los contemporáneos desarrollos teóricos como heréti$\cos y$ "modernistas", sino que se erigen con la intención de ocultar y consolidar las intenciones de las principales instituciones religiosas de conseguir en el poder político, beneficios para acentuar su esfera de acción; todo esto, bajo un mensaje que pretendía ser religioso pero que encubría ambiciones de poder, en vez de sentidos e interrogantes por cuestiones políticas más que existenciales. Este desarrollo, llevado a cabo por las principales autoridades eclesiásticas (aunque no por todas) junto con la respuesta conflictiva que suponen las propuestas analíticas, son en gran medida responsables de una lectura instrumentalista que se realiza sobre el fenómeno religioso, reduciéndolo puramente a un ámbito político o a un ámbito doctrinal, sin plantearse cuestiones inherentes al fenómeno específico y que denotan características esenciales de concepción y manifestación, no solamente de la sociedad en su desarrollo histórico, sino también del hombre en su situación existencial.

Una nueva perspectiva del fenómeno religioso surge junto con las primeras críticas a la Modernidad. Desde el vaticinio nietzscheano de la muerte de Dios, los grandes relatos, entre ellos el discurso de la Modernidad, empiezan a desmoronarse uno tras otro. La pregunta "chay una racionalidad universal y común a todos los seres humanos cualquiera sea su diversidad étnica, lingüística, cultural $[y]$ religiosa?" (Palacio 2006, 51), que caracterizaba a las tesis filosóficas de la Ilustración, empieza a carecer de sentido al no poder sostenerse, ya que la concepción de "un sujeto único, idéntico, razón universal, sede de la certeza y de la verdad ab- soluta" (Castro 2002 citado en Palacio 2006, 51). El impulso que le brinda, a ese desmoronamiento, el holocausto y la Segunda Guerra Mundial plantean los interrogantes de si las promesas hechas tiempo atrás por la Razón y la Libertad deberían desarrollarse de un modo diferente del que llevó al caos del dominio de la razón instrumental o caso contrario, superarse aceptando que el "gran relato ha perdido su credibilidad, sea cual sea el modo de unificación que se le haya asignado: relato especulativo, relato de emancipación" (Lyotard 1991, 32). A este proceso de crisis y replanteamiento, Hopenhayn lo analiza como una profundización del espíritu secular moderno. A tal fin, sostiene:

En este sentido, la nueva oleada secularizadora constituye una radicalización de la potencia desmitificadora de la modernidad. Ya no solo se impugna, en el sentido clásico de la secularización, el teocentrismo o el prejuicio moral. Ahora queda bajo la luz de la sospecha todo discurso totalizador para aprehender el mundo, $y$ todo Gran Razón para arbitrar las reglas del conocimiento y de la acción humanas. La secularización radical adquiere doble filo: por una parte libera al sujeto de todo relato que obstruya su poder para redefinirse a discreción y construir su propia visión de mundo; pero por otro lado sumerge a ese mismo sujeto en la orfandad que dicha libertad supone (1997, 13).

Esta ruptura (o profundización) de la cosmovisión moderna, donde el pensamiento trascendente variaba entre la teología y la Razón (Hopenhayn 1997), va a suponer también la crítica progresiva a perspectivas totalizadoras respecto del fenómeno religioso y cada vez más, la ampliación a la valoración y el estudio de lo religioso como un hecho universal, complejo y constitutivo del devenir históricocultural del ser humano.

En este contexto, los aportes realizados frente a la religión por la Antropología van a ser la génesis de una estimación cultural y plural. Abandonando de a poco el etnocentrismo generado por la búsqueda de la característica 
única y universal del sujeto, la apreciación de la diversidad cultural tiene sus primeros manifiestos en los abordajes realizados en primer lugar, por Frazer y posteriormente, por Malinowski y Radcliffe-Brown, quienes contribuyen a una difusión inicial de experiencias religiosas en los ambientes académicos, valorizando así, al fenómeno religioso para el mundo de los estudios culturales. Por su parte, la Sociología, con los desarrollos de Durkheim y Weber, brinda los primeros marcos teóricos para el análisis sociológico de la religión.

La dinámica histórico-cultural encuentra sus principales desarrollos de la mano de Rudolf Otto y Mircea Eliade. Otto va a marcar, en Alemania, las principales directrices del estudio cultural de la religión. Expresando las características de su tiempo, va a dar cuenta de la crisis del cimiento racional de nuevo siglo y los elementos que quedan frente a dicha crisis. Para Otto, la religión no es ni un elemento meramente irracional de las sociedades atrasadas que aún no han alcanzado una comprensión racional del desarrollo social, ni tampoco una manifestación doctrinal que deba necesariamente acatarse y seguir cual regla impuesta. Sin embargo, como religioso, da lugar a la cualidad vivencial y experimental del fenómeno religioso que delimita, una vez más, quienes están capacitados o no para abordar el estudio $y$ el entendimiento de la religión como experiencia humana. Sostiene este autor, la religión "nace de la convergencia de fuerzas racionales e irracionales, que permiten aislar lo sagrado como categoría aparte; es lo que se presenta como misterioso, fascinante, "completamente otro"” (Cipriani 2011, 174).

Esta concepción, lo "completamente otro", es retomada, en parte, por algunos de los representantes de la Escuela de Frankfurt. Si bien, el fenómeno religioso abordado por esta corriente mantiene fuertemente concepciones heredadas de su percepción marxista y se aborda a la religión, no como elocuente del sentido y la interpretación humana de su condición existencial, sino principalmente como institución que busca hacer uso de la razón instrumental para la domesticación del sujeto social (Horkheimer y Adorno 1998); puede verse cierta aproximación a través de la noción "nostalgia de lo Otro", que Horkheimer (1969, citado en Cipriani 2011) destaca sobre Adorno en sus estudios. Por ello, en ocasión de la muerte de su amigo, Adorno sostiene que este "ha hablado siempre de la nostalgia de lo Otro sin utilizar nunca la palabra cielo, eternidad, belleza o algo similar" (Cipriani 2011, 192) Y más adelante continua:

...yo creo, y esto es lo grandioso de la problemática, que él, interrogándose sobre el mundo, en definitiva ha considerado lo 'Otro', aunque estaba convencido de que este 'Otro' no es posible comprenderlo describiéndolo sino interpretando el mundo tal cual es $y$ considerando que el mundo no es lo único, no es la meta en donde pueden encontrar reposo nuestros pensamientos (192).

Lo "Otro" es tomado, analizado y comparado en Mircea Eliade. Su magno desarrollo dentro del campo de la Historia de las Religiones aporta la caracterización contextual en donde los mitos o la experiencia religiosa estudiada, se desarrollan y significan. Retomando a menudo la teoría de los arquetipos, más de Eugenio d'Ors que de Carl G. Jung (Diez de Velasco 2009), Eliade le brinda al estudio de las religiones una perspectiva cultural e histórica que permite un acercamiento desde el ámbito académico y una valoración del fenómeno como parte íntegra del devenir constructivo de las sociedades y como manifestación elocuente de los procesos culturales, de formas diferentes según el tiempo y el espacio, pero pertenecientes a una naturaleza en común: la naturaleza humana.

Ahora bien, a esta hermenéutica para el estudio del fenómeno religioso que se desarrolla en la primera mitad del siglo xx, se le suma con el desarrollo de los trabajos interdisciplinares, un abordaje complejo y dinámico que permite la integración plena del fenómeno religioso en el ámbito de los estudios culturales. Este campo de estudio, sostiene Palacio, es un espacio "tendiente a la superación de los marcos disciplinares en pos de una comprensión más amplia de la realidad social, cada vez más compleja y dinámica" (2011b, 129). A su vez, 
respecto de la característica de inter y transdisciplinariedad que adquieren estos estudios, argumenta que:

... no son paradigmas ni aspiran a ser nuevas disciplinas, sino que son tendencias académicas de estudio e investigación que se han producido en ciertos espacios del campo científico como un modo novedoso de convergencia de disciplinas $y$ perspectivas teóricas, antes aisladas, para tratar la cuestión candente de la organización simbólica de la vida urbana y las diversas relaciones de poder que se dan en una cultura situada (2011b, 129-130).

Para completar la cita, se aclara que estas tendencias sirven para analizar la vida simbólica del ser humano y sus creaciones culturales, no solo en la vida urbana, sino que también en todas las manifestaciones desarrolladas fuera de este contexto, como así también las relaciones que entre los diferentes ámbitos se establecen.

Esta complejidad, es la que el fenómeno religioso presenta en los nuevos estudios culturales. Una complejidad que no la reduce, en ningún momento, ni a una caracterización típica de una sociedad particular diferenciada de otras, ni a una manifestación doctrinal del carácter ontológico de la realidad, sino que la aborda como un ámbito de creación, re-significación y consumo cultural, dada universalmente pero manifestada con particularidades específicas del lugar y contexto donde emerge $y$ se desarrolla. La religión como fenómeno complejo de las sociedades contemporáneas no puede ser reducida a ningún ámbito particular, pero tampoco caracterizada en un ámbito específico. Retomando los aportes de Habermas, Taylor, Butler y West (2011), reconocer el poder que la religión tiene en la esfera pública es lograr un análisis no solo de la constitución del fenómeno religioso actual, sino también vislumbrar la complejidad de los ámbitos de una cultura que se constituyen y re-significan entre sí, como lo político, lo religioso, lo social y lo económico.

La apertura que generan los estudios interdisciplinares permiten realizar una lectura más colindante con la realidad social que lo estudios decimonónicos no brindaban. Entender a la religión como parte de este proceso de resignificación que plantea la cultura actual, da lugar al análisis de las manifestaciones culturales actuales desde una óptica holística o si se quiere, interdisciplinar para comprender mejor la amplitud de lo analizado.

Un ejemplo de temática factible para los estudios culturales actuales sobre el fenómeno religioso es la cuestión de las identidades. En el mundo actual donde predomina la industria cultural y los mensajes mediáticos tienen un carácter masivo, las identidades son conformadas:

... a partir de variadas pertenencias grupales e institucionales y múltiples interacciones y contactos, facilitados por el acceso mediático asignos urbanos de identificación. Las identidades de grupos $y$ colectivos, como procesos que se construyen a lo largo de la vida de las personas, son producto de narraciones sociales con que los grupos se auto-representan $y$ se presentan a los otros en el seno de cada cultura (Palacio 2011a, 155).

Esas narraciones sociales se enmarcan específicamente en cánones de valores que más allá de la caída del proyecto moderno, siguen siendo occidentales. Esto abre una veta de análisis al relacionarlo específicamente con la religión en su carácter institucional, que integra aristas políticas, culturales, económicas y sentimentales. Al respecto, Diez de Velasco argumenta:

Se producen, por tanto, movimientos de oposición a los cambios uniformadores que encuentran un material sensible justamente en los argumentos religiosos, que pueden detonar los mecanismos de insatisfacción al generar imaginarios modelos ideales que enfrentar a las miserias bien tangibles de la modernidad. Esta oposición frontal se suele denominar fundamentalismo $y$ en el mundo actual es uno de los modos más potentes de combinación de identidad y religión $(2005,165)$. 
Este ámbito de análisis que conjuga identidad y religión, es particularmente relevante al momento de iniciar un estudio sobre problemáticas del mundo actual que no pueden ser soslayadas, como la situación en Gaza, el imperialismo norteamericano, la resistencia de los grupos fundamentalistas islámicos, el rol de la sociedad islámica frente a estos grupos, el avance sionista y el rol del resto del pueblo judío frente al fundamentalismo, entre otros. Cada uno amerita un desarrollo particular, pero se citan aquí como modo de ejemplificación de una de las tantas posibilidades de análisis en los estudios culturales actuales con relación al fenómeno religioso.

\section{CONCLUSIÓN}

Retomando las principales conclusiones a las que se ha arribado en el desarrollo del artículo, es relevante decir, en primer lugar, que la naturaleza de la experiencia religiosa es variada y compleja. El intento de sistematizar y comunicar las características fenoménicas de dicha experiencia es siempre un acto vano, porque está en su naturaleza misma, abrir al humano a la comunicación con lo esencial más allá de lo aparente. Asimismo, esta naturaleza de la experiencia religiosa como variada y compleja que se la identificó, es susceptible de ser racional en parte, pero no es enteramente racional como lo afirma Pikaza (1999). Así, la reducción de la experiencia religiosa al ámbito meramente racional que se le hace desde el pensamiento contemporáneo, se debe más a una pretensión meta-lingüística del pensamiento racional que a la naturaleza misma de la experiencia religiosa; la cual expresa su naturaleza compleja, a través del símbolo, como un lenguaje meta-racional.

En segundo lugar, este tránsito de un estudio meramente fenomenológico a la valoración de la experiencia en su significación históricocultural es visible en el desarrollo progresivo que se da en su estudio, desde la consolidación de los primeros ámbitos hasta la situación actual. De una relación de lo religioso frente a las instituciones que intentaban cercenar la experiencia, el conocimiento y la difusión del mismo, ha tenido una evolución que tiende cada vez más a la apertura, al diálogo y a la comunicación para la construcción y la transmisión de la importancia que la religión presenta en las sociedades actuales. La búsqueda de sentido, los interrogantes esenciales y el recuerdo de la muerte, se constituyen y reconocen como ámbitos culturales propios de los hombres en su producción, circulación y consumo de sentidos y significantes de los cuales, la religión se presenta como la principal depositaria.

Por su parte, cercenar el estudio de lo religioso sin incluir su esfera (no solo) política, (no solo) teológica o (no solo) social, impide comprender de una manera más acertada los dinámicos procesos a los que se está sometidos contemporáneamente como sociedad. Así, los nuevos supuestos epistemológicos a los que los estudios culturales referencian posibilitan una valoración del fenómeno religioso y su rol en la sociedad, contribuyendo a superar concepciones instrumentalistas sobre el estudio de la religión.

\section{BIBLIOGRAFÍA}

LIBROS

Allen, Douglas. 1985. Mircea Eliade y el fenómeno religioso. Madrid, España: Ediciones Cristiandad.

Apel, Karl Otto. 1985. La transformación de la filosofía. Tomo I. Análisis del lenguaje, semiótica y hermenéutica. Madrid, España: Taurus Ediciones sa.

Cipriani, Roberto. 2011. Manual de sociología de la religión. Buenos Aires, Argentina: Siglo xxi.

Diez de Velasco, Francisco. 2005. La historia de las religiones: métodos y perspectivas. Madrid, España: Akal.

García Canclini, Néstor. 1995. Consumidores y ciudadanos. Conflictos multiculturales de la globalización. México: Grijalbo.

García Canclini, Néstor. 2004. Diferentes, Desiguales y Desconectados. Mapas de la interculturalidad. Barcelona, España: Gedisa.

Habermas, Jürgen, Charles Taylor, Judith Butler y Cornel West. 2011. El poder de la religión en la esfera pública. Madrid, España: Trotta. 
Horkheimer, Max y Theodor Adorno. 1998. Dialéctica de la Ilustración. Fragmentos filosóficos. España: Trotta.

Hopenhayn, Martín. 1997. Después del Nihilismo. De Nietzsche a Foucault. España: Editorial Andrés Bello.

Jaspers, Karl. 1968. Origen y meta de la Historia. Madrid, España: Revista de Occidente sa.

Lyotard, Jean-François. 1991. La condición postmoderna. Informe sobre el saber. Madrid, España: Cátedra.

Palacio, Marta. 2011a. "Problemas filosóficos de las identidades culturales". En Identidad, culturas, imaginarios. La Argentina del Bicentenario: una realidad para pensar también teológicamentem editado por la Sociedad Argentina de Teología, 153-164. Buenos Aires: San Benito.

Pikaza, Xabier. 1999. El fenómeno religioso. Curso fundamental de religión. Madrid, España: Trotta.

Taboada, Hernán. 1998. El símbolo en el pensamiento de Mircea Eliade. México:
Consejo Nacional de Ciencia y Tecnologia de México (соNacyt). http://www.cialc. unam.mx/ensayo/pdf/Simn_pensm.pdf.

PUBLICACIONES PERIÓDICAS

Diez de Velasco, Francisco. 2009. "Mircea Eliade y Eugenio d'Ors". Archaevs. Études d'Histoire des Religions 13: 227279.

Palacio, Marta. 2011b. "La interdisciplinariedad en los estudios sobre la ciudad". Artificium. Revista Iberoamericana de Estudios Culturales y Análisis Conceptual 2, n. 2: 126-140.

Palacio, Marta. 2006. "Los derechos de los rostros. Derechos humanos, liberalismo y exclusión". Anatellei 2: 51-62.

Fecha de ingreso: 18/08/2014

Fecha de aprobación: 20/03/2015 
